\title{
Stercoral Perforation of the Colon during Pregnancy: A Case Report and Review of the Literature
}

\author{
Anthony B. Costales, MD ${ }^{1}$ Amit K. Agarwal, MD ${ }^{2}$ Suneet P. Chauhan, MD 3 Jerrie S. Refuerzo, MD \\ Ethan A. Taub, $\mathrm{DO}^{2}$ \\ ${ }^{1}$ Department of Obstetrics and Gynecology and Reproductive \\ Medicine, The University of Texas Medical School at Houston, \\ Houston, Texas \\ 2 Department of Surgery, The University of Texas Medical School at \\ Houston, Houston, Texas \\ ${ }^{3}$ Department of Maternal Fetal Medicine, The University of Texas \\ Medical School at Houston, Houston, Texas \\ Am J Perinatol Rep 2015;5:e25-e29.

\begin{abstract}
Address for correspondence Anthony B. Costales, MD, Department of Obstetrics and Gynecology and Reproductive Medicine, 6431 Fannin Street, Suite 3.214, Houston, TX 77030

(e-mail: anthony.b.costales@uth.tmc.edu).
\end{abstract}

\begin{abstract}
Keywords

- colonic perforation

- pregnancy

- stercoral perforation

Stercoral perforation of the colon, though rare, is associated with high mortality. Review of the literature identified only three prior cases reported during pregnancy. We report a case on a multiparous female presenting at 31 weeks of gestation with acute abdominal pain. Computed tomography suggested a sigmoid colon perforation. An urgent exploratory laparotomy was performed where feculent peritonitis and a stercoral perforation of the sigmoid colon was confirmed. A cesarean delivery and sigmoid colectomy with descending end colostomy was performed. While the newborn had an uncomplicated course, the mother developed an intra-abdominal abscess requiring operative management.
\end{abstract}

Stercoral perforation of the colon, defined as perforation resulting from pressure necrosis of a fecal mass, is rare, but can be life-threatening, with reported mortality as high as $47 \%{ }^{1}$ Through 2014 , only three cases during pregnancy have been reported in the English language. ${ }^{2}$ We report a case of stercoral perforation occurring during pregnancy, with an uneventful outcome for the infant, but postoperative morbidity in the mother.

\section{Case Report}

A 38-year-old woman, gravida 6, para 2, at 31 weeks and 2 days of gestation presented to an outside hospital emergency department with acute onset of severe periumbilical pain. The pain developed over 3 days and subsequently radiated to the right lower quadrant following significant straining during a bowel movement. She also reported nausea and vomiting for 2 days. Her medical history included chronic constipation, with bowel movements occurring once every 2 weeks, a history of methamphetamine abuse, one previous cesarean delivery for placental abruption, bipolar disorder, chronic hypertension, and a previous open cholecystectomy. Her medications included olanzapine, and sublingual buprenorphine. Her pregnancy had been uneventful except for persistence of constipation, for which stool softeners, cathartics, and enemas were of minimal relief.

Upon presentation to the outside facility, the patient's temperature was $37.0^{\circ} \mathrm{C}$, heart rate 100 beats per minute, respiratory rate $22 / \mathrm{min}$, but blood pressure was not reported. Fetal heart tones were noted to be category I and without contractions on tocometry. Physical examination was notable for a distended and tender abdomen with mild rebound tenderness. Laboratory findings revealed a leukocytosis (white blood cells: $26.8 \times 10^{3} / \mathrm{mm}^{3}$ ) with a left shift (neutrophils: received

July 15, 2014

accepted after revision

November 28, 2014

published online

March 4, 2015
DOI http://dx.doi.org/

10.1055/s-0034-1544105. ISSN 2157-6998.
Copyright $\odot 2015$ by Thieme Medical Publishers, Inc., 333 Seventh Avenue, New York, NY 10001, USA. Tel: +1(212) 584-4662.
License terms

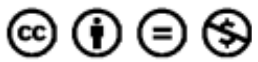


86\%) and a bandemia (bands: $12 \%$ ), and mild anemia (hematocrit: 33\%). Fetal ultrasound showed an anterior placenta with no suggestion of placenta previa or abruption. Given the above constellation of findings, the primary working diagnosis was acute appendicitis.

A right lower quadrant ultrasound revealed moderate free fluid within the right lower quadrant, but the appendix could not be definitively identified. Magnetic resonance imaging of the abdomen without contrast identified minimal-likely physiologic-free fluid within the bilateral paracolic gutters and stool throughout the colon. There were multiple borderline distended loops of small bowel without evidence of obstruction or ileus. The proximal aspect of the appendix could not be readily identified, however the distal two-thirds of the appendix was morphologically normal. The patient received intravenous fluids, an enema, $2 \mathrm{~g}$ of cefazolin, $2 \mathrm{~g}$ of ampicillin, two doses of $12 \mathrm{mg}$ of betamethasone, and six doses of $50 \mathrm{mg}$ of meperidine and $25 \mathrm{mg}$ of promethazine.

The patient was subsequently transferred to our tertiary care institution for clinical management and higher level of care. Upon arrival, her body temperature was $36.8^{\circ} \mathrm{C}$, heart rate was 118 beats per minute, blood pressure 159/82 mm Hg with oxygen saturation $98 \%$ on room air. Physical examination was notable for a distended, tender abdomen, with focal tenderness in right lower and upper quadrants. There were no bowel sounds present and a mass was palpable in the right upper quadrant, approximately $5 \mathrm{~cm} \times 5 \mathrm{~cm}$. On pelvic examination, the cervix was closed and the vaginal vault was without any evidence of blood or rupture of membranes. Fetal heart rate was 150 beats per minute with minimal variability, and without accelerations or decelerations. There were no contractions on tocometry. Bedside ultrasound examination revealed multiple dilated loops of bowel (-Fig. 1) an anterior placenta and a fetus in cephalic presentation. Laboratory data were significant for a normal white

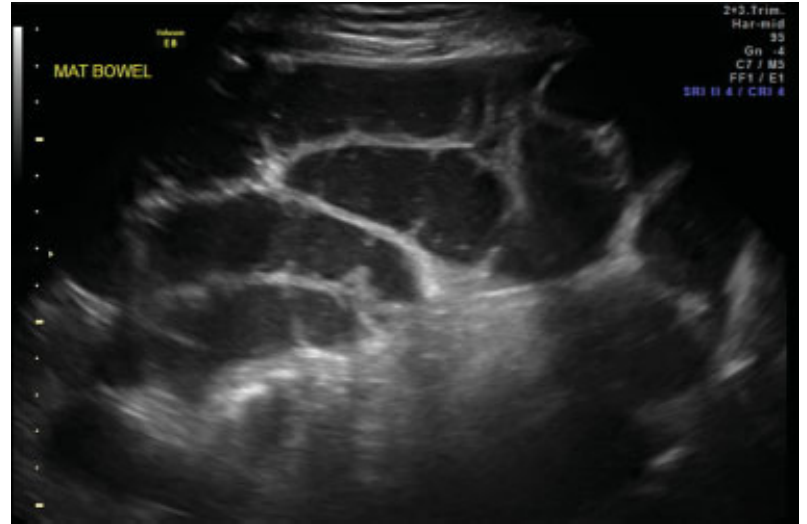

Fig. 1 Bedside abdominal ultrasound: Image shows multiple dilated loops of small bowel.

blood cell count of $5.900 \mathrm{~mm}^{3}$, bandemia of $20 \%$, mild anemia with a hematocrit of $35 \%$, and a urinary drug screen positive for opiates.

Approximately 2 hours after admission, the patient acutely desaturated to 89 to $91 \%$ on room air with correction only to $93 \%$ on $5 \mathrm{~L}$ nasal cannula, became tachycardic, and developed a fever of $38.0^{\circ} \mathrm{C}$. Computed tomographic angiogram was negative for acute pulmonary embolism. Computed tomography of the abdomen and pelvis with oral and intravenous contrast revealed moderate colonic distension, with the distal transverse and descending colon collapsed, and a significant amount of extraluminal air adjacent to the sigmoid colon within the sigmoid mesentery, suggesting a sigmoid colon perforation (-Fig. 2). Given these findings, an emergent laparotomy with cesarean delivery was performed.

On entering the peritoneal cavity, purulent ascites and a large amount of feces was encountered posterior to the uterus with fibrinous adhesions seen (-Fig. 3). This was surrounding an approximate $10 \mathrm{~cm}$ defect in the sigmoid colon. Given

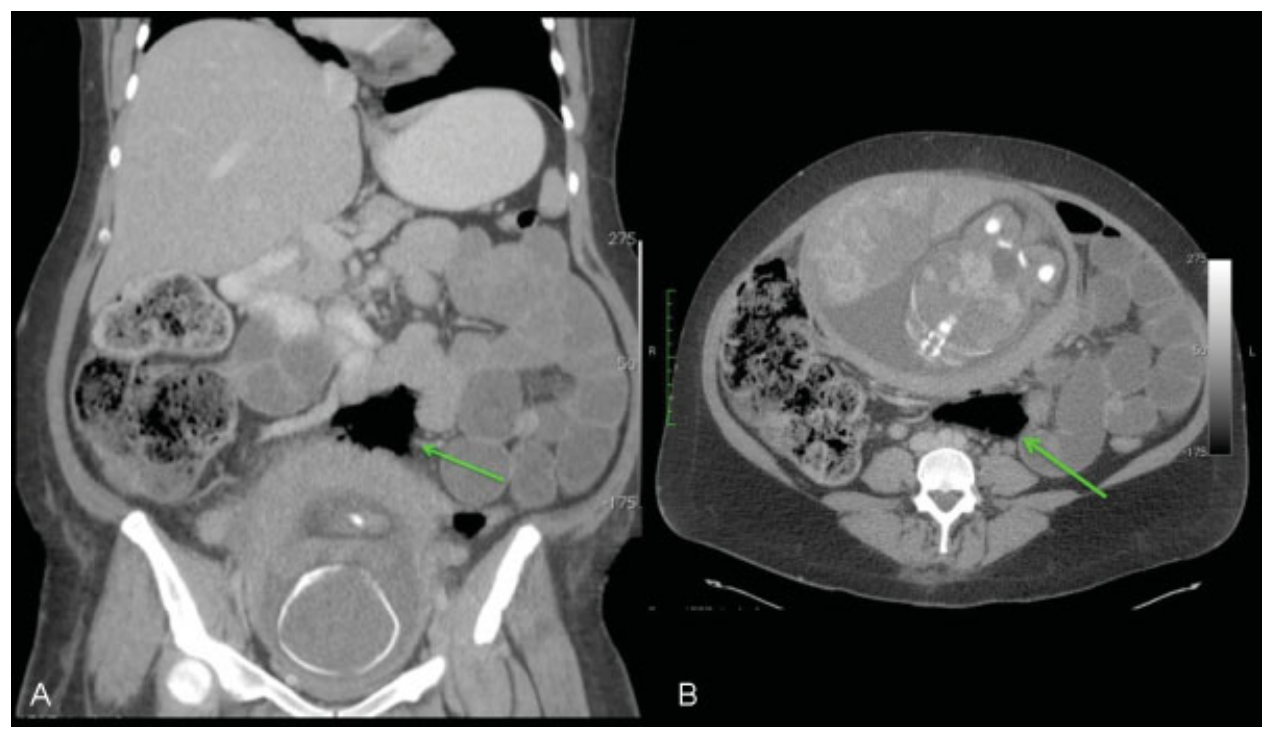

Fig. 2 Abdominopelvic computed tomography: Coronal (A) and transverse (B) sections showing extraluminal air (arrow) within the mesentery adjacent to the sigmoid colon suggesting colonic perforation. 


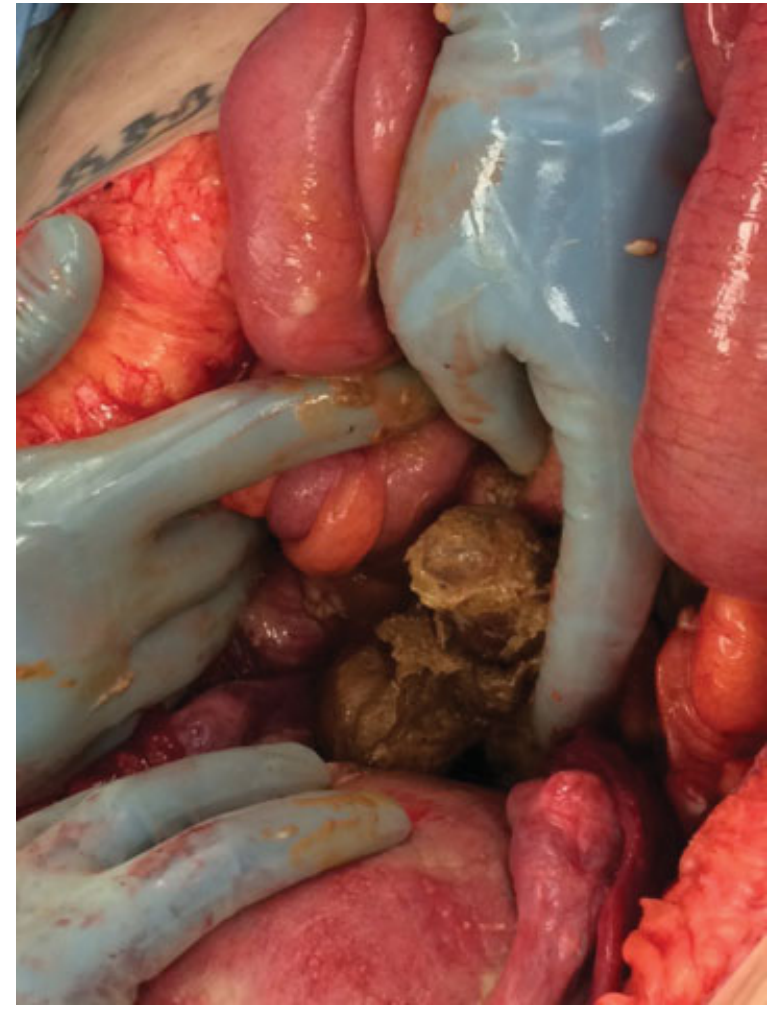

Fig. 3 Intraoperative findings: Feculent peritonitis with white fibrinous adhesions were noted on the small bowel and the posterior uterus. Multiple dilated loops of bowel were encountered.

the high risk for sepsis due to the finding of feculent material in the peritoneal cavity and the difficulty in performing a colonic resection in the setting of a gravid uterus, the decision was made to perform a repeat low transverse cesarean section. A sigmoid colectomy with descending end colostomy was then performed, the abdomen was copiously irrigated and the fascia was closed, but the skin was left open with application of negative wound therapy. The infant's Apgar score was 5 at 1 minute and 7 at 5 minutes with a pH of 7.11 and base excess of $-8 \mathrm{mmol} / \mathrm{L}$. Pathologic evaluation of the wall of the resected sigmoid colon showed acute inflammation with a $6 \mathrm{~cm} \times 5 \mathrm{~cm}$ perforation (-Fig. 4). Placental pathology was unremarkable.

The patient's postoperative course was complicated by an intra-abdominal abscess and fascial dehiscence. On postoperative day 6 , interventional radiology attempted computed tomography (CT)-guided abscess drainage, but ultimately on postoperative day 8 she returned to the operating room for repeat exploratory laparotomy, washout, and fascial closure with a monofilament synthetic absorbable suture. She was discharged home on postoperative day 25 afebrile, normotensive, nontachycardic, with a patent and productive stoma. The infant by the 2 nd postoperative day was stable on room air and was tolerating tube feeds. She was given a prophylactic course of antibiotics given the maternal sepsis, but the infant was without evidence of infection. The infant is planned to be discharged at approximately 5 weeks of life.

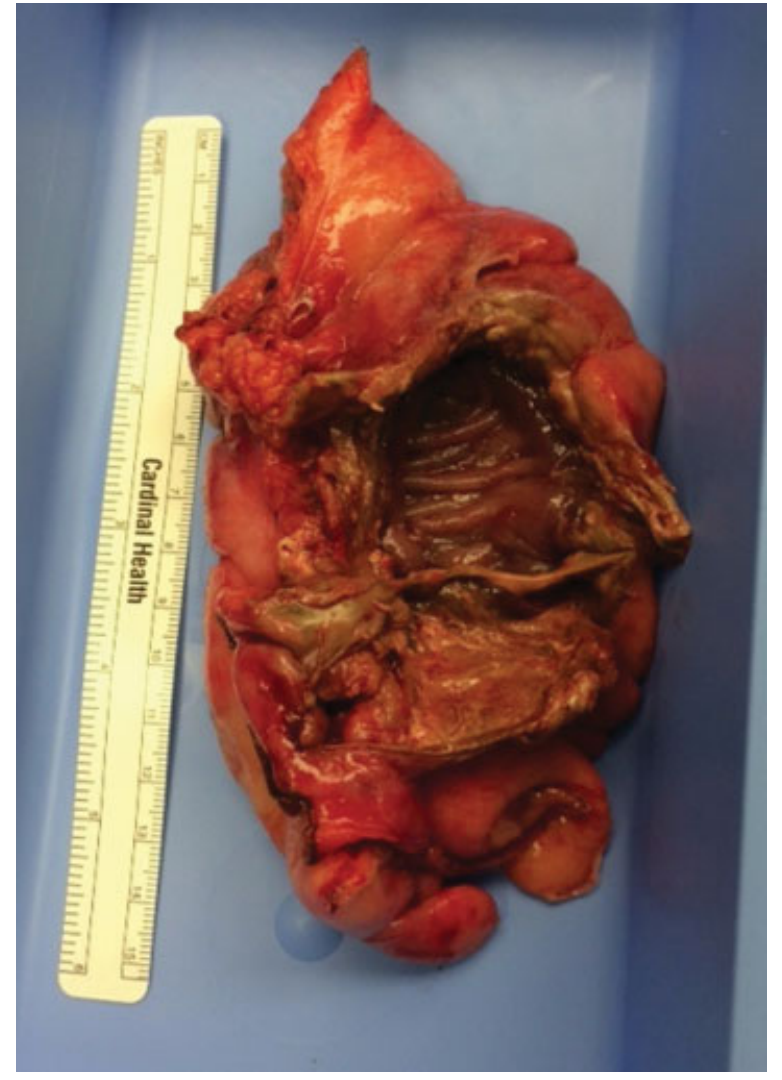

Fig. 4 Sigmoid colon: Gross pathologic specimen showing an ovoid perforation of the sigmoid colon measuring approximately 6 $\mathrm{cm} \times 4 \mathrm{~cm}$, consistent with stercoral perforation.

\section{Comment}

The first case of stercoral perforation was reported in $1894 .^{1}$ A review by Chakravartty et al identified only 217 cases since $1894 .^{3}$ It is commonly seen in the elderly with the median age of presentation is 62 years, ranging from 20 to 86 years. ${ }^{4}$

Maurer et al proposed diagnostic criteria which included: (1) round or ovoid perforation extending more than $1 \mathrm{~cm}$ in diameter and on the antimesenteric border of the bowel; (2) fecalomas present within the colon, protruding through the perforation site, or lying within the peritoneal cavity; and (3) pressure necrosis or ulcer with chronic inflammatory reaction around the perforation site on microscopic examination. ${ }^{4}$ Cases with any other colonic pathology including Hirschsprung disease, carcinoma, and diverticulitis need to be excluded. In our case, there was a $6 \mathrm{~cm}$ ovoid perforation in the sigmoid colon with feculent peritonitis, and microscopically, there was inflammation noted in the wall at the site of the perforation, meeting all three of the criteria proposed by Maurer et al.

Stercoral perforation is associated with chronic constipation in $81 \%$ of the patients with the site of perforation occurring in the sigmoid or rectosigmoid in $71 \%$ of the cases. ${ }^{4}$ There is a known association of constipation with chronic use of opiates, nonsteroidal anti-inflammatory drugs, antacids, tricyclic antidepressants, hypothyroidism, diabetic enteropathy, and hemiparesis. ${ }^{2,3}$ 
The pathogenesis of this disease is complicated, but the proposed mechanism is as follows: (1) fecalomas cause narrowing of the distal colon resulting in higher intraluminal pressure; (2) this exceeds the capillary perfusion pressure of the bowel wall and thus leads to pressure necrosis, especially on the antimesenteric border; (3) the blood supply of the sigmoid colon is diminished relative to the rest of the colon, predisposing to necrosis, eventually leading to ulceration and finally perforation., ${ }^{5,6}$ Our patient had a long standing history of constipation beginning 3 to 4 years before her pregnancy and a history of substance abuse, which she admitted had worsened during the pregnancy. She was taking sublingual buprenorphine, for a reported history of methamphetamine abuse, however constipation with buprenorphine has been shown to be less frequent than with morphine use and in a study of sublingual administration of buprenorphine in the elderly, constipation was reported in $<2 \%$ of the patients. ${ }^{7}$

Stercoral perforation in pregnancy is extremely uncommon, with our case being only the fourth reported. In one case, the patient was at 41 weeks gestation and presented with a history of clay pica, chronic constipation, and severe abdominal pain and subsequently delivered a stillborn infant and the mother died shortly after delivery. Stercoral perforation was only diagnosed on autopsy. ${ }^{8}$ In a subsequent case, the patient presented with severe abdominal pain at 36 weeks gestation. Given an unclear diagnosis, a cesarean delivery was performed and the patient delivered a live infant, but stercoral perforation was found intraoperatively. Mother and infant were discharged home on the 5th postoperative day without incident. ${ }^{9}$ The final case was of a patient at 22 weeks gestation who presented with severe abdominal pain and history of constipation was initially triaged with an abdominal X-ray and diagnosed with a threatened miscarriage. Her symptoms continued and she was ultimately diagnosed by an abdominal CT to have a perforation. She was taken for an emergent colonic resection, but 5 hours following the conclusion of the laparotomy, the patient delivered the infant vaginally, with the neonate dying almost immediately after delivery. ${ }^{2}$ Our case differs from the three previous published cases on pregnant women with stercoral perforation in that our patient was not discharged on initial presentation, but remained hospitalized, and multiple nondiagnostic imaging studies were performed, delaying diagnosis.

Acute abdominal pain in pregnancy is a diagnostic conundrum. The differential diagnosis is vast and the clinical evaluation is challenging given the gravid uterus. However, the tenants for evaluation of a patient with acute abdominal pain requiring possible surgical intervention in pregnancy should be similar to the nonpregnant patient. Imaging in pregnancy is a common area of hesitancy because of concerns with radiation; however the risks and benefits must be weighed, including delaying the diagnosis and increasing maternal morbidity, as well as fetal morbidity.

In this case, the patient was presumed to have acute appendicitis, but there was a delay in diagnosis as these imaging modalities have limitations in visualizing the appendix. The normal appendix is rarely identified in pregnancy, and nonvisualization on ultrasound cannot reliably exclude appendicitis. ${ }^{10}$ CT has a 94 to $98 \%$ sensitivity and a $98 \%$ specificity in accurately diagnosing acute appendicitis outside of pregnancy, ${ }^{11,12}$ but also has a high negative predictive value in pregnancy at $99 \% .{ }^{13}$ Although ultrasound and magnetic resonance imaging are useful in patients with presumed intestinal perforation, they are not considered definitive in diagnosing such conditions. ${ }^{14}$ Regarding the diagnosis of bowel perforation, computed tomography is the imaging modality of choice with a sensitivity as high as $85.5 \%$ in patients outside of pregnancy. ${ }^{15}$ With respect to imaging and radiation exposure in pregnancy, the American College of Obstetrics and Gynecology (ACOG) released a committee opinion in 2004 describing guidelines for diagnostic imaging. The fetal exposure to a CT scan of the abdomen and pelvis is $\sim 3.5 \mathrm{rad}$, which is less than the $5 \mathrm{rad}$ that has been possibly associated with an increase in fetal anomalies or pregnancy loss if between 8 and 15 weeks gestation. ${ }^{16}$ However, at 31 weeks, 1 to 2 rad of fetal exposure may increase the risk of leukemia by a factor of 1.5 to 2.0 over the baseline incidence that is estimated at 1 in $2,000 .^{16}$

In a patient presenting with acute abdominal pain in pregnancy, with a history of chronic constipation, intestinal perforation, specifically stercoral perforation, should be considered in the differential diagnosis. With mortality as high as $47 \%,{ }^{1}$ this puts both the mother and fetus at high risk of morbidity. Prompt diagnosis and treatment is the best way to prevent a poor outcome in a patient with stercoral perforation, and it begins with clinical suspicion. We conclude that although this clinical condition is rare, stercoral perforation should be considered in women with chronic constipation and an acute abdomen.

\section{Conflict of Interest}

The authors have no conflicts of interest to disclose.

\section{References}

1 Serpell JW, Nicholls RJ. Stercoral perforation of the colon. Br J Surg 1990;77(12):1325-1329

2 Matsushita T, Yumoto Y, Fukushima K, et al. Stercoral perforation of the colon during pregnancy. J Obstet Gynaecol Res 2011;37(11): $1685-1688$

3 Chakravartty S, Chang A, Nunoo-Mensah J. A systematic review of stercoral perforation. Colorectal Dis 2013;15(8):930-935

4 Maurer CA, Renzulli P, Mazzucchelli L, Egger B, Seiler CA, Büchler MW. Use of accurate diagnostic criteria may increase incidence of stercoral perforation of the colon. Dis Colon Rectum 2000;43(7): 991-998

5 Kumar P, Pearce O, Higginson A. Imaging manifestations of faecal impaction and stercoral perforation. Clin Radiol 2011;66(1): 83-88

6 Hsiao TF, Chou YH. Stercoral perforation of colon: a rare but important mimicker of acute appendicitis. Am J Emerg Med 2010;28(1):112.e1-112.e2

7 Likar R. Transdermal buprenorphine in the management of persistent pain - safety aspects. Ther Clin Risk Manag 2006;2(1): 115-125

8 Russell WL. Stercoraceous ulcer. Am Surg 1976;42(6):416-420 
9 Sung JF, Salvay HB, Hansman MF, Taslimi MM. Stercoral perforation of the colon with favorable pregnancy outcome. Obstet Gynecol 2009;113(2 Pt 2):491-492

10 Israel GM, Malguria N, McCarthy S, Copel J, Weinreb J. MRI vs. ultrasound for suspected appendicitis during pregnancy. J Magn Reson Imaging 2008;28(2):428-433

11 Furukawa A, Sakoda M, Yamasaki M, et al. Gastrointestinal tract perforation: CT diagnosis of presence, site, and cause. Abdom Imaging 2005;30(5):524-534

12 Rao PM, Rhea JT, Novelline RA, Mostafavi AA, McCabe CJ. Effect of computed tomography of the appendix on treatment of patients and use of hospital resources. N Engl J Med 1998;338(3): $141-146$
13 Lazarus E, Mayo-Smith WW, Mainiero MB, Spencer PK. CT in the evaluation of nontraumatic abdominal pain in pregnant women. Radiology 2007;244(3):784-790

14 Singh JP, Steward MJ, Booth TC, Mukhtar H, Murray D. Evolution of imaging for abdominal perforation. Ann R Coll Surg Engl 2010; 92(3):182-188

15 Maniatis V, Chryssikopoulos H, Roussakis A, et al. Perforation of the alimentary tract: evaluation with computed tomography. Abdom Imaging 2000;25(4):373-379

16 ACOG Committee Opinion No. ACOG Committee Opinion. Number 299, September 2004 (replaces No. 158, September 1995). Guidelines for diagnostic imaging during pregnancy. Obstet Gynecol 2004;104(3):647-651 